\title{
Skolem Mean Like Labeling
}

\author{
V. Shendra Shainy, V. Balaji
}

\begin{abstract}
Let $G=(V, E)$ be a graph of order $p$ and size $q$. A graph $G$ is said to be a skolem mean like graph if $p=q+1$. Here we prove the theorems to study the characterization for path and star. We prove that the path $p_{a}=p_{1} \wedge p_{2} \wedge \cdots \wedge p_{b}$ with $b-1$ wedges is a skolem mean like graph and if $a \geq 4, K_{1, a}$ is not a skolem mean like graph. Also we prove that the two star $K_{1, a} \wedge K_{1, b}$ is not a skolem mean like graph if and only if $|a-b| \leq 4$.
\end{abstract}

Keywords: Skolem mean like labeling, Skolem mean like graph, path and star.

\section{Introduction}

Let $G=(V, E)$ be a graph of order $p$ and size $q$. In [1],[2] and [3] the approach of skolem mean labeling was made familiar.The rule for a graph to be a skolem mean is that $p>q+1$. The theorems to study path, star and charecterization for the skolem mean labeling of two star is proved. We proved that any path is a skolem mean graph and $K_{1, m}$ is not a skolem mean graph for $m \geq 4$. Also we prove that the two star $K_{1, m} \cup K_{1, n}$ is a skolem mean graph if and only if $|m-n| \leq 4$.

\section{Pre requisities}

Definition 2.1. A graph $G=(V, E)$ with $p$ nodes and $q$ links is said to be a skolem mean like graph if there exists a function $f$ from the node set of $G$ to $\{1,2, \ldots, p\}$ such that the induced map $f^{*}$ is defined by

Manuscript received October 3, 2017; accepted June 25, 2018.

V. Shendra Shainy, V. Balaji are with the Department of Mathematics, Sacred Heart College, Tirupattur635601, India 


$$
f^{*}(e=g h)=\left\{\begin{array}{ccc}
\frac{f(g)+f(h)}{2} & \text { if } f(g)+f(h) \text { is even } \\
\frac{f(g)+f(h)+1}{2} & \text { if } f(g)+f(h) \text { is odd }
\end{array}\right.
$$

then the resulting links get unique labels $\{2,3, \ldots, p\}$.

Definition 2.2. A wedge is defined as a bridge connecting two components of a graph, denoted as $\wedge, \omega(G \wedge)<\omega(G)$.

$K_{1, m} \cup K_{1, n}$ is a two star and is a two component or a disconnected graph, Whereas, $K_{1, m} \wedge$ $K_{1, n}$ is a two star but a connected graph which means adding a wedge to a disconnected graph with two components becomes a connected or a single component graph with three components and two wedge becomes a connected or a single component graph.

Note: The only graph satisfying the rule $p=q+1$ is skolem mean like graph.

\section{Main Results}

Theorem 3.1. Any path $P_{a}=\left(P_{1} \wedge P_{2} \wedge P_{3} \ldots, \wedge P_{b}\right)$ with $(b-1)$ wedge is a skolem mean like graph.

Proof. Let $P_{a}$ be the path with links $u_{1} u_{2}, u_{2} u_{3}, \ldots, u_{a-1} u_{a}$.

Define a map $f: V\left(P_{a}\right) \rightarrow\{1,2, \ldots, p\}$ by $f\left(\alpha_{u}\right)=u$ for $1 \leq u \leq a$.

Then the respective label of the link $\alpha_{u} \alpha_{u+1}$ is $u+1$ for $1 \leq u \leq a-1$.

Hence $P_{a}$ is a skolem mean like graph.

Now we are going to study the existence of skolem mean like labeling of stars. We observe that some stars are having skolem mean like labeling and some stars are not having skolem mean like labeling, as the star $K_{1,1}$ is $P_{1}$ and $K_{1,2}$ is $P_{3}$ are skolem mean like graphs by theorem (3.2).

Theorem 3.2. $K_{1, a}$ is not a skolem mean like graph, if $a \geq 4$.

Proof. Suppose $K_{1, a}$ is a skolem mean like graph, then the unique link labels must be $\{2,3, \cdots . a+1\}$.Let $\left(V_{1}, V_{2}\right)$ be the bipartition of $K_{1, a}$ with $V_{1}=\{u\}$. To get the link label $a+1$, we must have $a+1$ and $a$ as the node labels of adjacent node. Thus either $a+1$ or $a$ must be the label of $u$. In both cases, it is not possible to label the vertices without labeling three of them as consecutive integers in the component $G$. Then, the respective induced link labels are two of them will be same. Therefore the induced link labels of $G$ are not unique, which contradicts our supposition. Hence it is proved that $K_{1, a}$ is not a skolem mean like graph. 
Now we find the charecterization for the skolem mean like labeling for two star graph.

Theorem 3.3. The two star $K_{1, a} \wedge K_{1, b}$ is a skolem mean like graph if and only if $|a-b| \leq 4$

Proof. Without loss of generality, we consider that $a \leq b$.

Let us first take the case $|a-b| \leq 4$.

There are five cases Viz. $b=a, b=a+1, b=a+2, b=a+3$ and $b=a+4$. We have to prove that $K_{1, a} \wedge K_{1, b}$ is a skolem mean like graph for the above cases.

Case 1: Let $b=a$.

Consider the graph $G=K_{1, a} \wedge K_{1, b}=K_{1, a} \wedge K_{1, a}=2\left(K_{1, a}\right)$. Let $\{\alpha\} \cup\left\{\alpha_{u}: 1 \leq u \leq a\right\}$ and $\{\beta\} \cup\left\{\beta_{v}: 1 \leq v \leq a\right\}$ be the node set of first component and second component of $K_{1, a}$ respectively. Then, $G$ has $2 a+1$ links and $2 a+2$ nodes.

We have $V(G) \rightarrow\{\alpha, \beta\} \cup\left\{\alpha_{u}: 1 \leq u \leq a\right\} \cup\left\{\beta_{v}: 1 \leq v \leq a\right\}$.

The appropriate node labeling $V(G) \rightarrow\{1,2, \ldots, 2 a+2\}$ is defined as follows:

$f(\alpha)=3 ; f(\beta)=2 a+1$.

$f\left(\alpha_{u}\right)=2 u \quad$ for $\quad 1 \leq u \leq a-1$

$f\left(\alpha_{a}\right)=1$

$f\left(\beta_{v}\right)=2 v+3 \quad$ for $\quad 1 \leq v \leq a-2$

$f\left(\beta_{a-1}\right)=2 a$

and

$f\left(\beta_{a}\right)=2 a+2$.

The respective link labels are given below :

The link label of $\alpha \alpha_{u}$ is $u+2$ for $1 \leq u \leq a-1$ and $\beta \beta_{v}$ is $a+v+2$ for $1 \leq v \leq a-2$. Also, the link label of $\alpha \alpha_{a}$ is 2; the wedge label of $\alpha_{u} \beta_{v}$ is $a+2$ for all $\alpha_{u}$ and $\beta_{v}$ such that $\frac{f\left(\alpha_{u}\right)+f\left(\beta_{v}\right)+1}{2}=a+2 ; \beta \beta_{a-1}$ is $2 a+1$ and $\beta \beta_{a}$ is $2 a+2$.Therefore, the appropriate link labels of $G=\{2,3, \ldots, a+1, a+2, a+3, \ldots, 2 a, 2 a+1,2 a+2\}$

Therefore, the number of distinct label is $((2 a+2)-2+1))=2 a+1$ unique links.

Case 2: Let $b=a+1$.

Consider the graph $G=K_{1, a} \wedge K_{1, b}=K_{1, a} \wedge K_{1, a+1}$. Let $\{\alpha\} \cup\left\{\alpha_{u}: 1 \leq u \leq a\right\}$ be the nodes of $K_{1, a}$ and $\{\beta\} \cup\left\{\beta_{v}: 1 \leq v \leq a+1\right\}$ be those $K_{1, a+1}$. Then $G$ has $2 a+3$ nodes and $2 a+2$ links.

We have $V(G)=\{\alpha, \beta\} \cup\left\{\alpha_{u}: 1 \leq u \leq a\right\} \cup\left\{\beta_{v}: 1 \leq v \leq a+1\right\}$

The appropriate node labeling $f: V(G) \rightarrow\{1,2 \ldots, 2 a+3\}$ is defined as follows :

$f(\alpha)=2 ; f(\beta)=2 a+2$.

$f\left(\alpha_{u}\right)=2 u-1 \quad$ for $\quad 1 \leq u \leq a$

$f\left(\beta_{v}\right)=2 v+2 \quad$ for $\quad 1 \leq v \leq a-1$

$f\left(\beta_{a}\right)=2 a+1 \quad$ and

$f\left(\beta_{a+1}\right)=2 a+3$. 
The respective link labels are given below :

The link label of $\alpha \alpha_{u}$ is $u+1$ for $1 \leq u \leq a$ and $\beta \beta_{v}$ is $a+v+2$ for $1 \leq v \leq a-1$. Also, the wedge label of $\alpha_{u} \beta_{v}$ is $a+2$ for all $\alpha_{u}$ and $\beta_{v}$ such that $\frac{f\left(\alpha_{u}\right)+f\left(\bar{\beta}_{v}\right)+1}{2}=$ $a+2 ; \beta \beta_{a}$ is $2 a+2$ and $\beta \beta_{a+1}$ is $2 a+3$. Therefore, the appropriate link labels of $G=\{2,3, \ldots, a+1, a+2, a+3, \ldots, 2 a+1,2 a+2,2 a+3\}$

Therefore, the number of unique label is $((2 a+3)-2+1))=2 \mathrm{a}+2$ unique links.

Case 3: Let $b=a+2$

Consider the graph $G=K_{1, a} \wedge K_{1, b}=K_{1, a} \wedge K_{1, a+2}$. Let $\{\alpha\} \cup\left\{\alpha_{u}: 1 \leq u \leq a\right\}$ be the nodes of $K_{1, a}$ and $\{\beta\} \cup\left\{\beta_{v}: 1 \leq v \leq a+2\right\}$ be those $K_{1, a+2}$. Then $G$ has $2 a+4$ nodes and $2 a+3$ links.

We have $V(G)=\{\alpha, \beta\} \cup\left\{\alpha_{u}: 1 \leq u \leq a\right\} \cup\left\{\beta_{v}: 1 \leq v \leq a+2\right\}$

The appropriate node labeling $f:(V(G)) \rightarrow\{1,2, \ldots, 2 a+4\}$ is defined as follows :

$$
\begin{array}{lll}
f(\alpha)=1 ; f(\beta)=2 a+3 . & & \\
f\left(\alpha_{u}\right)=2 u & \text { for } 1 \leq u \leq a \\
f\left(\beta_{v}\right)=2 v+1 & \text { for } 1 \leq v \leq a \\
f\left(\beta_{a+1}\right)=2 a+2 & \text { and } & \\
f\left(\beta_{a+2}\right)=2 a+4 . & &
\end{array}
$$

The respective link labels are given below :

The link label of $\alpha \alpha_{u}$ is $u+1$ for $1 \leq u \leq a$ and $\beta \beta_{v}$ is $a+v+2$ for $1 \leq v \leq a$;

Also, the wedge label of $\alpha_{u} \beta_{v}$ is $a+2$ for all $\alpha_{u}$ and $\beta_{v}$ such that $\frac{\left.f\left(\alpha_{u}\right)+f\left(\overline{\beta_{v}}\right)+1\right)}{2}=$ $a+2 ; \beta \beta_{a+1}$ is $2 a+3$ and $\beta \beta_{a+2}$ is $2 a+4$.

Therefore, the appropriate link labels of

$G=\{2,3, \ldots, a+1, a+2, a+3, \ldots, 2 a+2,2 a+3,2 a+4\}$

Therefore, the number of unique link label is $((2 a+4)-2+1))=2 a+3$ unique links.

Case 4: Let $b=a+3$

Consider the graph $G=K_{1, a} \wedge K_{1, b}=K_{1, a} \wedge K_{1, a+3}$. Let $\{\alpha\} \cup\left\{\alpha_{u}: 1 \leq u \leq a\right\}$ be the nodes of $K_{1, a}$ and $\{\beta\} \cup\left\{\beta_{v}: 1 \leq v \leq a+3\right\}$ be those $K_{1, a+3}$. Then $G$ has $2 a+5$ nodes and $2 a+4$ links.

We have $V(G)=\{\alpha, \beta\} \cup\left\{\alpha_{u}: 1 \leq u \leq a\right\} \cup\left\{\beta_{v}: 1 \leq v \leq a+3\right\}$

The appropriate node labeling $f:(V(G)) \rightarrow\{1,2, \ldots, 2 a+5\}$ is defined as follows : $f(\alpha)=1 ; f(\beta)=2 a+4$.
$f\left(\alpha_{u}\right)=2 u+1$
for $\quad 1 \leq u \leq a$
$f\left(\beta_{v}\right)=2 v$
for $1 \leq v \leq a+1$
$f\left(\beta_{a+2}\right)=2 a+3$
and
$f\left(\beta_{a+3}\right)=2 a+5$. 
The respective link labels are given below :

The link label of $\alpha \alpha_{u}$ is $u+1$ for $1 \leq u \leq a$ and $\beta \beta_{v}$ is $a+v+2$ for $1 \leq v \leq a+1$.

Also, the wedge label of $\alpha_{u} \beta_{v}$ is $a+2$ for all $\alpha_{u}$ and $\beta_{v}$ such that $\frac{f\left(\alpha_{u}\right)+f\left(\bar{\beta}_{v}\right)+1}{2}=$ $a+2 ; \beta \beta_{a+2}$ is $2 a+4$ and $\beta \beta_{a+3}$ is $2 a+5$. Therefore, the appropriate link labels of $G=\{2,3, \ldots, a+1, a+2, a+3, \ldots, 2 a+3,2 a+4,2 a+5\}$

Therefore the number of unique link label is $((2 a+5)-2+1))=2 a+4$ unique links.

Case 5: Let $b=a+4$.

Consider the graph $G=K_{1, a} \wedge K_{1, b}=K_{1, a} \wedge K_{1, a+4}$. Let $\{\alpha\} \cup\left\{\alpha_{u}: 1 \leq u \leq a\right\}$ be the nodes of $K_{1, a}$ and $\{\beta\} \cup\left\{\beta_{v}: 1 \leq v \leq a+4\right\}$ be those $K_{1, a+4}$. Then $G$ has $2 a+6$ nodes and $2 a+5$ links.

We have $V(G)=\{\alpha, \beta\} \cup\left\{\alpha_{u}: 1 \leq u \leq a\right\} \cup\left\{\beta_{v}: 1 \leq v \leq a+4\right\}$

The appropriate node labeling $f: V(G) \rightarrow\{1,2 \ldots, 2 a+6\}$ is defined as follows :

$$
\begin{aligned}
& f(\alpha)=2 ; \\
& f(\beta)=2 a+5 . \\
& f\left(\alpha_{u}\right)=2 u+2 \quad \text { for } \quad 1 \leq u \leq a \\
& f\left(\beta_{v}\right)=2 v-1 \quad \text { for } 1 \leq v \leq a+2 \\
& f\left(\beta_{a+3}\right)=2 a+4 \quad \text { and } \\
& f\left(\beta_{a+4}\right)=2 a+6 .
\end{aligned}
$$

The respective link labels are given below :

The link label of $\alpha \alpha_{u}$ is $u+2$ for $1 \leq u \leq a$ and $\beta \beta_{v}$ is $a+v+2$ for $1 \leq v \leq a+2$.

Also,the wedge label of $\alpha \beta_{1}$ is $2 ; \beta \beta_{a+3}$ is $2 a+5$ and $\beta \beta_{a+4}$ is $2 a+6$. Therefore, the appropriate link labels of

$G=\{2,3, \ldots, a+1, a+2, a+3, \ldots, 2 a+4,2 a+5,2 a+6\}$

Therefore the number of unique link label is $((2 a+6)-2+1))=2 \mathrm{a}+5$ unique links. Hence, the graph $G$ is skolem mean like graph if $|a-b| \leq 4$.

Conversely, let us consider the case $|a-b|>4$.

Suppose, that $G=K_{1, a} \wedge K_{1, b}$ for $b=a+r$ for $r \geq 5$ is a skolem mean like graph, let us assume that $G=G_{1} \wedge G_{2}$ for $G_{1}=K_{1, a+r}$ and $G_{2}=K_{1, a}$.

Let us now consider the case when $r=5$ and $a=1$, then the graph $G_{1,6} \wedge G_{1,1}$ have 9 nodes and 8 links.

Let $V(G)=\left\{u_{1, s}: 0 \leq s \leq 1\right\} \cup\left\{v_{2, s}: 0 \leq s \leq 6\right\}$ and

$E(G)=\left\{u_{1,0} u_{1,1}\right\} \cup\left\{v_{2,0} v_{2, s}: 1 \leq s \leq 6\right\} \cup$

$\left\{u_{1, h} v_{2, s}\right.$ : for any one of $\mathrm{h}$ and $\left.\mathrm{s}, 0 \leq h \leq 1 ; 0 \leq s \leq 6\right\}$.

Suppose $G$ is a skolem mean like graph,let $\mathrm{p}=|V|=9$ and $\mathrm{q}=|E|=8$.

Then, there exists a function $f$ from the node set of $G$ to $\{1,2, . ., p\}$ such that the induced the map $f^{*}$ is defined by 


$$
f^{*}(e=g h)=\left\{\begin{array}{ccc}
\frac{f(g)+f(h)}{2} & \text { if } & f(g)+f(h) \text { is even } \\
\frac{f(g)+f(h)+1}{2} & \text { if } & f(g)+f(h) \text { is odd. }
\end{array}\right.
$$

Then the links get unique labels $2,3, \ldots$ p. Let $t_{i, s}$ be the label given to the node $u_{i, s}$ for $0 \leq s \leq 1$ and $v_{2, s}$ for $0 \leq s \leq 6$ and $x_{i, s}$ be the respective link label of the link $u_{1,0}$ $u_{1,1}$ and $v_{2,0} v_{2, s}$ for $1 \leq s \leq 6$.

Case (a): $t_{2,0}=8$.

If $t_{2, s}=2 b-1$ and $t_{2, c}=2 b$ for some $\mathrm{b}$ and for some $\mathrm{s}, \mathrm{c}$ then, $f^{*}\left(v_{2,0} v_{2, s}\right)=\left[\frac{8+2 b-1}{2}\right]=b+4=\left[\frac{8+2 b}{2}\right]=f^{*}\left(v_{2,0} v_{2, c}\right)$.

This is not possible as $f^{*}$ is a bijection.

Therefore, the feasible node labels are (either 1 or 2), (either 3 or 4 ), (either 5 or 6 ), 7 and 9.

These five labels are not adequate to label the six nodes, $t_{2, s}$ for $1 \leq s \leq 6$.

Case (b): $t_{2,0}=7$

If $t_{2, s}=2 b+1$ and $t_{2, c}=2 b$ for some $\mathrm{b}$ and for some $\mathrm{s}, \mathrm{c}$ then,

$f^{*}\left(v_{2,0} v_{2, s}\right)=\left[\frac{7+2 b+1}{2}\right]=b+4=\left[\frac{7+2 b}{2}\right]=f^{*}\left(v_{2,0} v_{2, c}\right)$.

This is not possible as $f^{*}$ is a bijection.

Therefore, the feasible node labels are 1, (either 2 or 3), (either 4 or 5), 6 and (either 8 or 9 ).

These five labels are not adequate to label the six nodes, $t_{2, s}$ for $1 \leq s \leq 6$.

Case (c): $t_{2,0}=6$

If $t_{2, s}=2 b-1$ and $t_{2, c}=2 b$ for some $\mathrm{b}$ and for some $\mathrm{s}, \mathrm{c}$ then,

$f^{*}\left(v_{2,0} v_{2, s}\right)=\left[\frac{6+2 b-1}{2}\right]=b+3=\left[\frac{6+2 b}{2}\right]=f^{*}\left(v_{2,0} v_{2, c}\right)$.

This is not possible as $f^{*}$ is a bijection.

Therefore, the feasible node labels are (either 1 or 2 ), (either 3 or 4), 5, (either 7 or 8)

and 9.

These five labels are not adequate to label the six nodes, $t_{2, s}$ for $1 \leq s \leq 6$. 
Case (d): $t_{2,0}=5$

If $t_{2, s}=2 b+1$ and $t_{2, c}=2 b$ for some $\mathrm{b}$ and for some $\mathrm{s}, \mathrm{c}$ then,

$f^{*}\left(v_{2,0} v_{2, s}\right)=\left[\frac{5+2 b+1}{2}\right]=b+3=\left[\frac{5+2 b}{2}\right]=f^{*}\left(v_{2,0} v_{2, c}\right)$.

This is not possible as $f^{*}$ is a bijection.

Therefore, the feasible node labels are 1, (either 2 or 3), 4, (either 6 or 7 ) and (either 8 or 9 ).

These five labels are not adequate to label the six nodes, $t_{2, s}$ for $1 \leq s \leq 6$.

Case (e): $t_{2,0}=4$

If $t_{2, s}=2 b-1$ and $t_{2, c}=2 b$ for some $\mathrm{b}$ and for some $\mathrm{s}, \mathrm{c}$ then,

$f^{*}\left(v_{2,0} v_{2, s}\right)=\left[\frac{4+2 b-1}{2}\right]=b+2=\left[\frac{4+2 b}{2}\right]=f^{*}\left(v_{2,0} v_{2, c}\right)$.

This is not possible as $f^{*}$ is a bijection.

Therefore, the feasible node labels are (either 1 or 2), 3, (either 5 or 6), (either 7 or 8)

and 9.

These five labels are not adequate to label the six nodes, $t_{2, s}$ for $1 \leq s \leq 6$.

Case (f): $t_{2,0}=3$

If $t_{2, s}=2 b+1$ and $t_{2, c}=2 b$ for some $\mathrm{b}$ and for some $\mathrm{s}, \mathrm{c}$ then,

$f^{*}\left(v_{2,0} v_{2, s}\right)=\left[\frac{3+2 b+1}{2}\right]=b+2=\left[\frac{3+2 b}{2}\right]=f^{*}\left(v_{2,0} v_{2, c}\right)$.

This is not possible as $f^{*}$ is a bijection.

Therefore, the feasible node labels are 1,2, (either 4 or 5), (either 6 or 7 ) and (either 8 or 9 ).

These five labels are not adequate to label the six nodes, $t_{2, s}$ for $1 \leq s \leq 6$.

Case (g): $t_{2,0}=2$

If $t_{2, s}=2 b-1$ and $t_{2, c}=2 b$ for some $\mathrm{b}$ and for some $\mathrm{s}, \mathrm{c}$ then,

$f^{*}\left(v_{2,0} v_{2, s}\right)=\left[\frac{2+2 b-1}{2}\right]=b+1=\left[\frac{2+2 b}{2}\right]=f^{*}\left(v_{2,0} v_{2, c}\right)$.

This is not possible as $f^{*}$ is a bijection.

Therefore, the feasible nodes labels are 1, (either 3 or 4 ), (either 5 or 6),(either 7 or 8)

and 9.

These five labels are not adequate to label the six nodes, $t_{2, s}$ for $1 \leq s \leq 6$. 
Case (h): when $t_{2,0}=1$

If $t_{2, s}=2 b+1$ and $t_{2, c}=2 b$ for some $\mathrm{b}$ and for some $\mathrm{s}, \mathrm{c}$ then, $f^{*}\left(v_{2,0} v_{2, s}\right)=\left[\frac{1+2 b+1}{2}\right]=b+1=\left[\frac{1+2 b}{2}\right]=f^{*}\left(v_{2,0} v_{2, c}\right)$.

This is not possible as $f^{*}$ is a bijection.

Therefore, the feasible node labels are (either 2 or 3), (either 4 or 5 ), (either 6 or 7 ) and (either 8 or 9 ).

These four labels are not adequate to label the six nodes, $t_{2, s}$ for $1 \leq s \leq 6$.

Therefore, we clearly say that $G$ is not a skolem mean like graph by the above cases. Therefore, $G=K_{1,6} \wedge K_{1,1}$ is not a skolem mean like graph, when $|a-b|=5$.

Similarly, we can prove that $G=K_{1,7} \wedge K_{1,1}$ is not a skolem mean like graph, when $|a-b|=6$.

Hence, $G=K_{1, b} \wedge K_{1, a}$ is not a skolem mean like graph if $|a-b| \geq 5$.

\section{Suggestion for future work}

Further work can be carried out by the bounds of the three star and four star graphs. Also, to find $n$ generalizing the bounds of star graphs, that is to find the upper and lower bounds of $n$ star graph in skolem mean like labeling.

Acknowledgement Dr. V. Balaji acknowledges for financial assistance (No.FMRP5766 / 15(SERO/UGC))

\section{References}

[1] V. Balaji, D.S.T Ramesh, A. Subramanian A, Skolem Mean Labeling, Bulletin of Pure and Applied Sciences, vol. 26E No. 2,2007, $245-248$.

[2] J.A Gallian, A dynamic survey of graph labeling, The Electronic Journal of Combinatorics, 6(2010), \#DS6.

[3] F. HARARY, Graph Theory, Addison Wesley, Reading, 1969. 